\title{
A Performance Modeling and Evaluation of the Cambridge Fast Ring
}

\author{
Mirjana Zafirovic-Vukotic and Ignas G. Niemegeers, Member, IEEE
}

\begin{abstract}
The Cambridge Fast Ring (CFR) is a high speed slotted ring. The features that make it suitable for use at very large transmission rates are the synchronous transmission, the simplicity of the protocol, and the possibility of immediate retransmission of erroneous minipackets. The CFR allows for only one slot at a time to be used by each station. Performance of the CFR with normal slots is studied in this paper. We show that the CFR can be represented by a multiqueue multiple cyclic server model with a 1-limited service discipline and with a restriction that only one server at a time can be serving a queue. Exact necessary and sufficient stability conditions are stated. An approximate analytic $M|G| 1$ vacation model in which analysis concentrates on one station while the others are represented by a vacation period is developed to estimate the expected message waiting times. It is shown that the model is accurate and usable over a wide range of parameters. A performance evaluation of the CFR based on this model is presented. The performance of the CFR is compared to a variant which does not restrict the number of slots a station may simultaneously use.
\end{abstract}

Index Terms-High speed local area networks, multiple cyclic server model, performance evaluation, performance modeling, serial interconnection structure, slotted rings.

\section{INTRODUCTION}

T has been shown that slotted ring protocols are suitable for high speed local area networks, see e.g. [33]. They are characterized by synchronous transmission which provides ease of implementation, and by relatively short, fixed length, minipackets. Examples can be found in the Cambridge Fast Ring (CFR) (see [29] and [15]), the Cambridge Backbone Network [12], Orwell [8], FXNET [6], and Upperbus [11]. The CFR has been developed starting from a medium speed ring: the Cambridge Ring [16]. It implements acknowledgment at the MAC layer and offers the possibility of immediate retransmission of erroneous minipackets, which distinguishes it from other slotted ring protocols, e.g., Orwell and the Cambridge Backbone Network.

The performance of the basic access mechanism (AM) of the CFR is studied in this paper. We also refer to this basic access mechanism as the CFR access mechanism. In the model only the operation with so called normal slots is considered. The load is of the asynchronous type. Transmission rates in excess

Manuscript received August 24, 1989; revised November 6, 1990 and January 13, 1991.

M. Zafirovic-Vukotic was with University of Twente, Tele-Informatics and Open Systems Group, 7500AE Enschede, Holland. She is now with M.Pupin Institute, University of Belgrade, Belgrade, Yugoslavia.

I. G. Niemegeers is with University of Twente, Tele-Informatics and Open Systems Group, 7500AE Enschede, Holland.

IEEE Log Number 9200324. of $100 \mathrm{Mb} / \mathrm{s}$ are assumed. The model allows us to estimate the expected message delays. Only the queueing delays for access to the medium and the transfer delays are taken into account. Delays due to the processing of the messages are not included. One of the main questions is how the mechanism performs in different applications, e.g., integrated services local area networks, backbone networks, and interconnection networks in multicomputers.

We show that the CFR access mechanism can be represented by a multiqueue multiple cyclic server model with a 1-limited service discipline and with a restriction that only one server at a time can be serving a queue. Exact necessary and sufficient stability conditions are presented in this paper. Further, a Poisson bulk arrival process of minipackets is assumed. At the moment, exact solutions are only available in the literature for a single cyclic server case. An approximate analytical $\mathrm{M}|\mathrm{G}| 1$ vacation model is developed to derive the expected message waiting times. The analysis in the model concentrates on one station which is represented by a single server queue while the effect of all other stations is incorporated in a server vacation period. The model has been tested by extensive and detailed simulations in a number of cases expected to be realistic for high speed local area networks. It is shown that it is accurate and usable over a wide range of parameters and the model seems to be less accurate for asymmetric configurations with high loads. A performance evaluation of the CFR access mechanism has been done using the approximate model. The sensitivity to the number of active stations, the number of slots, the transmission rate, the expected message length, and the slot information field length has been studied. One of the questions which is also addressed is how the mechanism performs in different applications. Such performance evaluation of the CFR is not yet available in the literature.

We assume the reader is familiar with the terminology used to describe networks and protocols, e.g., protocol data unit (PDU), service data unit (SDU), medium access control (MAC), data link layer (DLL), and protocol control information (PCI). However, enough context information is provided to make the paper readable to people not directly involved in this area. A message corresponds to a Data Link SDU (D_SDU) and a minipacket corresponds to a MAC_PDU.

The paper is organized as follows. In Section II, the relevant features of the CFR protocol are presented. In Section III, the state of the art in performance analysis of the CFR is presented. In Section IV, notation is introduced. In Section V the workload model is presented. In Section VI the stability conditions are stated. In Section VII an $\mathrm{M}|\mathrm{G}| 1$ vacation model 
with bulk arrival process is presented. In Section VIII this model is tested by comparing to simulations. In Section IX, the performance evaluation of the access mechanism is described. Finally, in Section X some conclusions regarding the model and the performance of the CFR access mechanism are presented.

\section{The CAmbridge Fast Ring Access Mechanism}

Let us present the most relevant features of the CFR with regard to performance analysis. See [15] and [29] for more details.

The ring is partitioned into equal length slots (Fig. 1). We assume that this is achieved by introducing a latency register at the monitor station to virtually lengthen the ring to a multiple of the slot length. Slots circulate around the ring and can be empty or full. Messages are segmented and MAC PCI is added to form a number of minipackets. Each minipacket is sent to the destination separately.

A full slot is occupied by a minipacket. Stations are actively coupled to the ring: they repeat or modify the slots. An empty slot may be filled by a minipacket. A full slot circulating around the ring, reaches the destination station which reads it and passes it on to a higher layer. We assume that each station is capable of using every empty slot that arrives and of reading every slot destined to itself.

In the CFR, a station which has filled a slot (source station) also releases this after it has circulated around the ring. We call this technique source release. There are two types of slots: normal and channel slots. A normal slot after being used has to be emptied and must be passed on to the next downstream station which may use it (see Fig. 2 for an illustration of the functioning of a normal slot). A channel slot may be reused by the source. Only one slot at a time can be carrying minipackets from the same source.

Using source release and permitting only one slot at a time to be used by a station provides the opportunity of immediately retransmitting an erroneous minipacket when a negative acknowledgment is received by the source. This in turn implies that the sequencing of the minipackets is preserved. Other slotted ring protocols (e.g., Orwell) do not have this property. A fair access to the medium is guaranteed by using normal slots. Channel slots do not guarantee fairness. They are intended for large bandwidth users or bursty sources.

In this paper the performance analysis is done for a ring with normal slots only. Analytic modeling of the operation with both normal and channel slots would be much more complex. However, we did a simulative performance analysis for that case in [38].

\section{State of the ART of Performance ANALYSIS OF THE CAMBRIDGE FAST RING}

A number of analytic models have been developed for the slotted ring protocols, e.g., in [14], [17], [5], and [21]. However, these are either models of a slotted ring protocol where more than one slot at a time can be used by a station, or the workload, in particular the arrival process of minipackets, is based on interactive users. In high speed local area networks

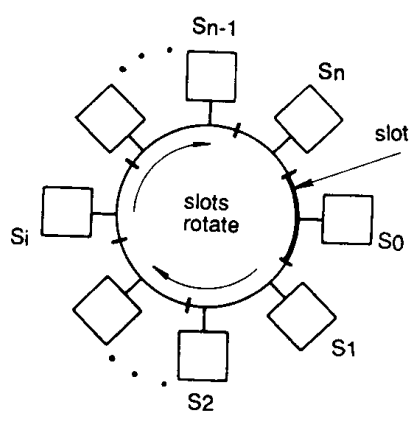

Fig. 1. The slotted ring structure.

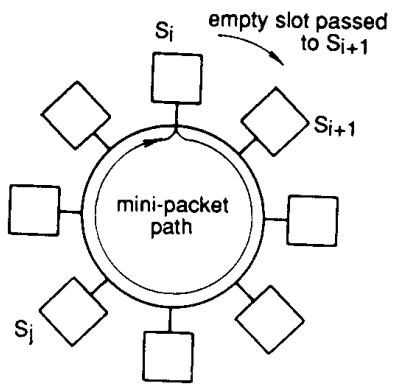
Fig. 2. Path of a minipacket sent from $S_{i}$ to $S_{j}$ in the CFR using a normal
slot.

however, high throughput users provide most of the load, not the interactive users. So, there is no appropriate analytic model available in the literature which can be used for the CFR.

For a complete overview of the state of the art and a short presentation of new models of the basic access mechanisms of the slotted ring protocols the reader is referred to [34]. Further, peer studies presenting new analytic models we developed for other slotted rings are presented in [36] and [37].

The performance of the slotted rings in high speed networks for telephone traffic only has been evaluated in [8], [9], and [22]. A study in [15] shows that the use of channel slots in CFR improves its maximum utilization. A study in [32] evaluates multiple Cambridge rings which consist of a number of rings coupled by bridges.

\section{NOTATION}

Let us introduce the following notation:

$n-$ the number of (active) stations minus one;

$S_{i}-i$ th station in the ring, $i=0,1, \cdots n$, and for simplicity of notation we assume that station $S_{0}$ can also be denoted as $S_{n+1}$;

$w$ - the transmission rate $(\mathrm{b} / \mu \mathrm{s}$ or $\mathrm{Mb} / \mathrm{s})$;

$\sigma-$ the duration of a slot $(\mu \mathrm{s})$;

$\nu$ - the duration of an information field of a slot $(\mu \mathrm{s})$, such that $\nu<\sigma$;

$\lambda_{i}$ - the message arrival rate at station $S_{i}\left(\mu \mathrm{s}^{-1}\right), i=$ $0, \cdots, n$; 
$Z_{i}$ - the random variable denoting the bulk size of the arrival process at $S_{i}$, i.e., the number of minipackets a message is split into, $i=0, \cdots, n$;

$\gamma_{i}, E Z_{i} 2$ - the first two moments of $Z_{i}, i=0, \cdots, n$;

$\rho$ - the relative load, or the expected number of minipackets arriving in the system during $\sigma$ time units, such that

$$
\rho=\sum_{i=0}^{n} \lambda_{i} \gamma_{i} \sigma
$$

$X_{i}$ - the random variable denoting a minipacket service time in the model $(\mu \mathrm{s}), i=0, \cdots, n$;

$E X_{i}, E X_{i}^{2}$ - the first two moments of $X_{i}, i=0, \cdots, n$;

$Y_{i}$ - the random variable denoting a message service time in the model $(\mu \mathrm{s}), i=0, \cdots, n$;

$E Y_{i}, E Y_{i}^{2}$ - the first two moments of $Y_{i}, i=0, \cdots, n$;

$E R$ - the expected cycle duration in a cyclic server model;

$p_{i j}$ - an element of the communication source_to_destination matrix, $\left\|p_{i j}\right\|_{n+1 \times n+1}$ which represents the relative traffic intensity from source $S_{i}$ to destination $S_{j}$, and

$$
0 \leq p_{i j} \leq 1, \text { and } \sum_{j=0}^{n} p_{i j}=1, \quad i, j=0, \cdots, n \text {. }
$$

$s$ - the number of slots in the ring;

$\tau_{i j}$ - the propagation time from $S_{i}$ to $S_{j}$ including the latency at station $S_{j}(\mu \mathrm{s}), i, j=0, \cdots, n$;

$\tau$ - the slot rotation time or $\tau_{i i}$ for all $i(\mu \mathrm{s})$, such that

$$
\tau=\sum_{i=0}^{n} \tau_{i, i+1}, \text { and } \tau=s \sigma
$$

with index $n+1$ referring to station $S_{0}$;

$\tau_{i}$ - the expected propagation time of a message or a minipacket sent by $S_{i}$ from $S_{i}$ to the destination $(\mu \mathrm{s})$, $i=0, \cdots, n$;

$E V_{i}$ - the expected minipacket waiting time, or the expected minipacket waiting time for access to the MAC service, i.e., in terms of the queueing model, the expected minipacket waiting time in the queue at $S_{i}$ until the beginning of its service $(\mu \mathrm{s}), i=$ $0, \cdots, n$;

$E W_{i}-$ the expected message waiting time, or the expected message waiting time of the first minipacket of a message for access to the MAC service, i.e., in terms of the queueing model the expected message waiting time from its arrival at $S_{i}$ until the beginning of its service $(\mu \mathrm{s}), i=0, \cdots, n$;

$E T_{i}$ - the expected message delay or the expected service time of a DLL service provider per message of $S_{i}$, i.e., in terms of the queueing model, the expected message delay from arrival at $S_{i}$ until its complete delivery at the destination $(\mu \mathrm{s}), i=0, \cdots, n$.

\section{WORKLOAD MODEL}

Let us now specify the workload model. It includes the arrival process of messages and of minipackets, the distribution of their lengths, and the traffic pattern in the ring.

We assume that messages arrive at the DLL layer of station $S_{i}$ according to a Poisson process with intensity $\lambda_{i}$. Messages are segmented and MAC protocol control information of nonzero length is added to form a number of minipackets. So, the arrival process of minipackets at $S_{i}$ can be considered to be a bulk Poisson process.

Message lengths are assumed to be independent and identically distributed (i.i.d.). The information field of a minipacket in the slotted ring protocol has a constant length $\nu \times w$ (bit). The protocol control information of a minipacket has a constant length too. So, the length of a minipacket is constant and equal to $\sigma \times w$ (bit), i.e., a slot length. The random variable $Z_{i}$ has a distribution which is derived from the message length distribution.

We allow each station to send to any other station including itself, i.e., $p_{i j}$ can take any value such that relations (2) hold.

\section{Stability Conditions}

\section{A. Exact Multiple Cyclic Server Presentation of the CFR}

One can exactly represent a slotted ring by a multiqueue cyclic polling model with $n+1$ queues and $s$ cyclic servers (Fig. 4). A minipacket corresponds to a customer in this model. The $i$ th queue is at $S_{i}$. Each server corresponds to a slot. A server is passed cyclically from $S_{i}$ to $S_{i+1}$. This corresponds to passing an empty slot from $S_{i}$ to $S_{i+1}$. The server switchover time from $S_{i}$ to $S_{i+1}$ is equal to $t_{i, i+1}$. When a queue that is visited by a server is not empty, the server first removes and then starts serving the oldest customer. In the slotted ring protocol, this corresponds to a situation where an empty slot arrives at $S_{i}$ and there is a minipacket to be sent, so the slot is filled with the oldest minipacket. The service time corresponds to the time a slot is full, i.e., to the ring delay. So, the service time is a constant and equal to the ring delay $\tau$. After processing one customer the server departs from the queue. Thus, the service discipline at the queue $S_{i}$ is a limited first-in-first-out discipline, with at most one customer served per visit to the queue (so called 1-limited service). The CFR permits only one slot at a time to be used by each station, i.e., a station can transmit its next minipacket only after it has released the slot used for the previous minipacket. Therefore in the model there is a restriction that only one server at a time can be serving each queue. Note that servers do not cycle independently of each other and that servers can pass each other. So, the CFR can be regarded as a multiqueue multiple cyclic server model with a 1 -limited service discipline and having at most one server at a time serving each queue.

\section{B. Patterns of Operation}

To find the stability conditions we analyze the system in the following two cases: 1) all the queues are unstable and 2) all the queues are stable. We first consider the case where all the queues are unstable, i.e., each station is empty with 
probability zero. Whenever a station already has a slot in use, it has to pass on empty slots unused even though it has minipackets queued up. Therefore, system capacity is wasted, which results in server overhead. The overhead depends on the pattern which the ring operation follows. Let us take a look at the best and worst performance patterns. In the best performance pattern it never happens that a station has a full slot in the ring when an empty slot arrives. So, after being released the empty slot visits the next station and serves it. In the worst performance pattern each slot after being released visits the next $s-1$ stations but cannot serve them since each of them is already using one of the other slots. Both patterns are such that if they are entered they will not be changed with probability one, provided all queues are unstable. This is due to the deterministic behavior if all the queues are nonempty and to the noncyclic character of the patterns. This analysis is also valid for the case of $m$ stations out of which $n$ stations are active $(n \leq m)$.

Fig. 3 illustrates the existence of a best and a worst performance pattern for the most simple case where this can occur: a CFR with 2 slots and 2 stations. The figure contains time-space diagrams showing when slots are occupied and when they are free. In both cases the system is initially completely empty. At a given moment both stations are turned on simultaneously and have a permanent backlog of minipackets from that moment on. Whether the ring settles in the best or the worst performance pattern depends on the moment the stations are turned on. In the best performance pattern the slot utilization is $2 / 3$, while in the worst performance pattern it is $1 / 2$.

Let us now look at the system when all queues are stable, so, each station is empty with a nonzero probability. In this case the system is randomly changing patterns of operation due to visiting empty stations. The patterns of operation and the corresponding quasistable states have been studied in the literature (e.g., [1], [3], and [4]). Some heuristic approximations of the bandwidth available per station when all queues are stable is given in [4]

We can now present a necessary condition and a sufficient condition for all the queues to be stable (Fig. 5). For determining the necessary condition we assume the best performance pattern for passing empty slots. For determining the sufficient condition we assume the worst performance pattern. This approach leads to the identification of two multiqueue multiple cyclic server models related to the CFR which differ in the switchover time and which are used to determine the two stability conditions.

\section{Necessary Condition}

Consider first the best performance pattern. So, each slot after being released is used by the first downstream station. In this case we can represent the system as a multiple cyclic server system with a limited service discipline in which the service time is equal to a slot rotation time, i.e., to the time a slot is occupied by a minipacket $(\tau)$, and the switchover time is equal to the duration of passing an empty slot to the first downstream station, i.e., $\tau /(n+1)$.

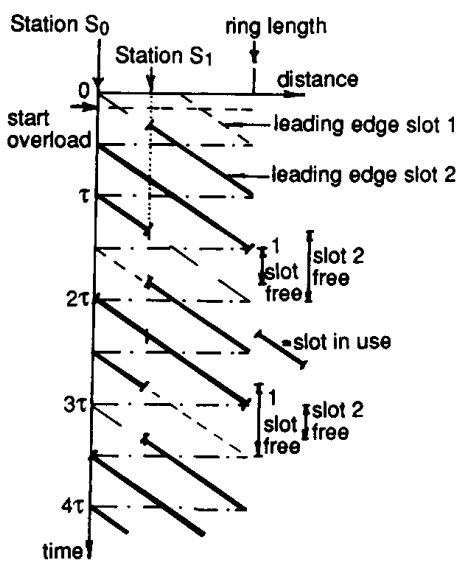

(a)

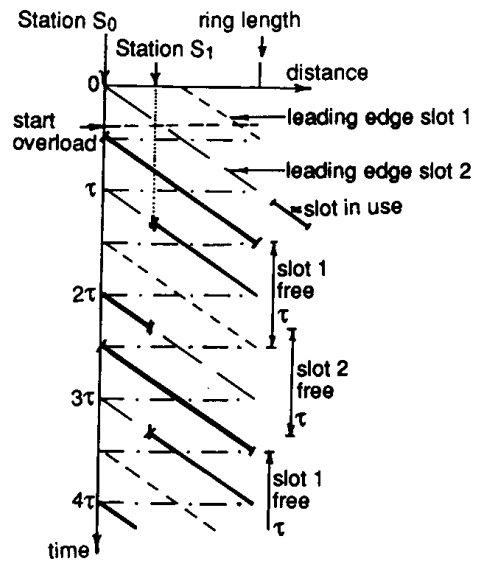

(b)

Fig. 3. (a) CFR under overload: best performance pattern-Case of 2 slots and 2 stations. (b) CFR under overload: worst performance pattern-Case of 2 slots and 2 stations.

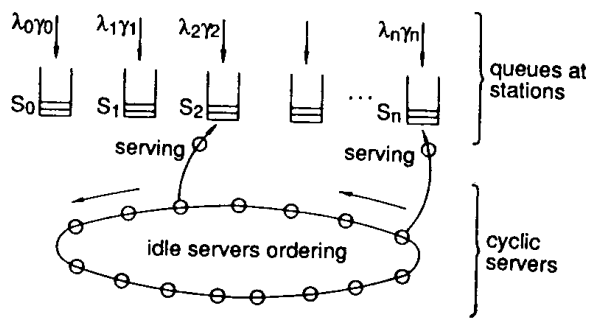

Fig. 4. A multiqueue multiple cyclic server model of a slotted ring.

A stability condition has been stated for a multiple cyclic server system (in [23]). It is based on the argument of the balance between the work which is arriving at the system and the work which can be done by the system. The same argument has been used for the case of a single cyclic server system in [19]. Applying this argument, we have the following necessary stability condition. Let $E R$ denote the expected server rotation time in this case. 


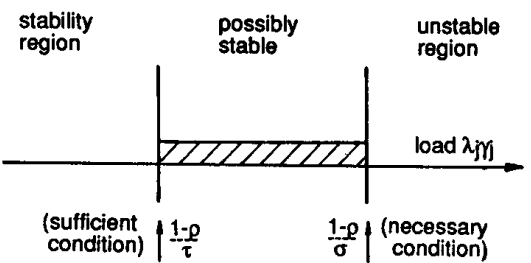

Fig. 5. An illustration of stable and instable regions with respect to the load at $S_{j}$.

If the system is stable, using the work balance argument, the following has been obtained:

$$
\begin{aligned}
E R & =\frac{\tau}{1-\frac{1}{s} \sum_{j=0}^{n} \lambda_{j} \gamma_{j} \tau} \\
& =\frac{\tau}{1-\rho}
\end{aligned}
$$

where $\rho$ is given in relation (1).

The necessary and sufficient condition for all queues to be stable, again using the same balance argument, is that for all $i$,

$$
\lambda_{i} \gamma_{i} E R<s, \quad i=0, \cdots, n .
$$

From (3), (4), and (5) it follows that

$$
\lambda_{i} \gamma_{i} \sigma<1-\rho, \quad i=0, \cdots, n .
$$

Note that the assumption $\rho<1$ which is necessary for $E R$ to be positive is implicit in relation (6).

\section{Sufficient Condition}

Assume now the worst case pattern. So, each slot after being emptied cannot be used by the next $s-1$ stations which it passes since they have other slots in use, i.e. they are being served by the other $s-1$ servers. In that case we can again identify a multiple cyclic server model with a 1-limited service discipline as follows. The service time is equal to a slot rotation time, i.e., to the time a slot is occupied by a minipacket $(\tau)$ and the switchover time is equal to the duration of passing an empty slot to the $s$ th downstream station, i.e., $s \tau /(n+1)$. In a similar way as for (6), one can propose the sufficient stability condition:

$$
\lambda_{j} \gamma_{j} \tau<1-\rho
$$

for each $j=0, \cdots, n$.

Note that if $s=1$ the necessary and the sufficient stability conditions are the same.

Further on we assume that all the queues are stable. Let us now analyze the stability conditions expressed in relations (6) and (7). For all the queues to be stable it is necessary that the arrival rate of minipackets at each $S_{i}$ per slot duration $\left(\lambda_{i} \gamma_{i} \sigma\right)$ is less than one minus the relative load $(\rho)$. For all the queues to be stable it is sufficient that the arrival rate of minipackets at each $S_{i}$ per ring latency $\left(\lambda_{i} \gamma_{i} \tau\right)$ is less than one minus the relative load. The relative load depends on the arrival rate of minipackets at each station $\left(\lambda_{i} \gamma_{i}, i=0, \cdots, n\right)$. This arrival rate depends on the arrival rate of messages at each station $\left(\lambda_{i}\right)$ and on the expected number of minipackets included in a message $\left(\gamma_{i}\right)$ which depends on the slot information field length $(\nu)$ and on the message length distribution. From (6) and (7), we have that the relative load or the total arrival rate of minipackets in $\sigma$ time units is bounded by 1 , if the system is stable.

\section{MODEL DESCRIPTION}

Let us now develop a model of the CFR access mechanism which estimates the expected message delay. There are models of similar systems available in the literature. An approximate model of the multiqueue multiple cyclic server system with a limited service discipline has been presented in [36]. Approximate analytic models for a single cyclic server system with a limited service discipline are given, e.g., in [10] and in [13, chapter 7] and an exact solution for a symmetric case, e.g., in [27]. However, we cannot use these results because they treat a single cyclic server case whereas we deal with a multiple cyclic server model and/or because of the restriction that each queue can be served by only one server at a time which introduces extra difficulties in providing estimates. These arguments do not hold if $s=1$, i.e., for a single slot case. For that case the reader is referred to [36]. In this section we develop an approximate server vacation model of the CFR.

\section{A. Concept of the Model}

An $M^{\mathrm{B}}|\mathrm{G}| 1$ vacation model with bulk arrivals of minipackets and individual service (corresponding to one minipacket transmitted per slot) can be used for approximate modeling of the queue at $S_{i}$. The model needs to take into account the occasional unavailability of the server at the queue due to slots being already in use. This is modeled by server vacations. Although it has been shown in Section VI that the CFR can be represented as a multiple cyclic server system (each server corresponding to a slot), a single server model (the server corresponding to the CFR MAC service provider) is used to actually estimate the message delays at the stations.

Assume that batches of minipackets arrive at $S_{i}$ according to a Poisson process with intensity $\lambda_{i}$ and with batch size $Z_{i}$.

Define the minipacket service time $X_{i}$ as the time between the following two instants: the time when a minipacket at $S_{i}$ begins transmission, and the arrival of the next empty slot that can be used by $S_{i}$. Service is offered to $S_{i}$ as long as the queue is not empty, and when the server finds the queue empty, it takes a vacation of duration $O_{i}$ (this is the exhaustive service from the operational model in cyclic service systems). Here, $O_{i}$ represents the time between two consecutive instants when service is available at $S_{i}$, provided that no customers are served between these two instants.

Note that this vacation period is different from the one in the vacation model representation of the single cyclic server system, where the server takes vacation to serve all other queues and only then it returns to $S_{i}$. Because of this we cannot compare our model with the approximate models of single cyclic server systems when represented as vacation models (for such models see [7], [25], and [28]). 
A companion model of this $\mathrm{M}^{\mathrm{B}}|\mathrm{G}| 1$ minipacket bulk arrival model can be made. It is an $M|G| 1$ model where a message rather than a minipacket is a service unit. It is straightforward to see that the distribution and moments of the message service time can be directly evaluated from the distributions of the batch size $Z_{i}$ and of the minipacket service time $X_{i}$. The model is illustrated in Fig. 6.

We assume that the number of nodes is larger than the number of slots. In case the number of slots is larger than the number of active stations $(k+1, k \leq n)$ in the ring, a part of the system capacity is wasted since at most $k+1$ slots can be used. We do not model this case. Therefore, we assume that all stations are active and that $s \leq n+1$. This is not an essential assumption and the model can be developed and extended for other cases as well. The assumption has been introduced mainly in order to simplify the presentation.

It is assumed in this model that the $X_{i}$ as well as the $O_{i}$ are i.i.d. and mutually independent. Because of this, queue lengths at all stations are also independent. It is further assumed that all the slots are independent, i.e., that the state of one slot does not give information about the state of other slots.

An $M^{\mathrm{B}}|\mathrm{G}| 1$ vacation model has been studied in [19] and [2]. However, as will be explained later on, we use an $M^{B}|G| 1$ model only to make a companion $\mathrm{M}|\mathrm{G}| 1$ vacation model and use the latter one for analysis. So, since it is not necessary we do not use the results for the bulk arrival case, except to determine the relationship between the expected message delay and the expected minipacket delay. An overview of $\mathrm{M}|\mathrm{G}| 1$ vacation models is available in [28].

In the following the model is presented. The probability $\pi_{i}$ that a slot arriving at $S_{i}$ cannot be used, given that $S_{i}$ is presently not using a slot, is evaluated first. Next, the distribution and the first two moments of the server vacation time $O_{i}$ and the minipacket service time $X_{i}$ are approximated. This determines the first two moments of the message service time, which in turn determines the mean message waiting time and the mean message delay.

\section{B. Probability $\pi_{i}$}

Let $\pi_{i}=\mathrm{P}$ a a slot arriving at $S_{i}$ is full $\mid S_{i}$ has no full slot in the ring\}. $\pi_{i}$ is used in estimates of the vacation and minipacket service time distributions. For the estimate of $\pi_{i}$ the reader is referred to the Appendix. Let us summarize the way it has been obtained.

$\pi_{i}$ has been estimated such that it includes the slot occupancy by a minipacket and the overhead due to the protocol. The protocol overhead refers to the propagation time (walk time) of empty slots between stations, while there are some minipackets waiting to be sent somewhere in the system. In terms of the multiple cyclic server model of the CFR, the servers in the cyclic server model are idling while there is work waiting in the queues. The protocol overhead is caused

- by empty slots visiting empty stations while there is work to be done in the system, i.e., there is a nonempty station waiting for an empty slot, and

- by empty slots visiting nonempty stations which are unable to use it since they have already a slot in use.

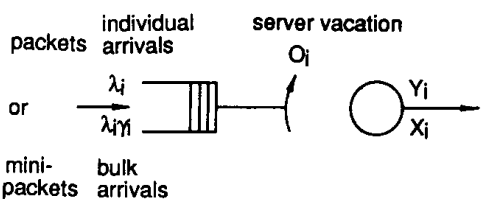

Fig. 6. A M $|\mathrm{G}| 1$ model with bulk arrivals of minipackets and server vacation periods.

The derivation of the estimate for $\pi_{i}$ makes use of the assumption of slot and queue length independence.

\section{Server Vacation Time and Minipacket Service Time}

Let us now evaluate the distribution of the vacation time $O_{i}$. A station which does not use an empty slot passes this slot to the first downstream station. After passing an empty slot, station $S_{i}$ will receive this particular slot back again after a period no more than $(n+1) \tau$. This is because there are $n$ other stations to be visited by this slot, and each can hold the slot for a maximum period $\tau$ equal to the time that a slot carries its minipacket. There is also a time $\tau$ spent being passed between stations, so we can be sure that the slot will return to $S_{i}$ after a period which does not exceed $(n+1) \tau$.

However, the maximum time between arrivals of an empty slot (not a particular one) at $S_{i}$ will be less. Since there are $s$ slots in the ring, the time between two arrivals of an empty slot at $S_{i}$ is not greater than the maximum for a particular slot, minus the duration of the other $(s-1)$ slots, $(n+1) \tau-(s-1) \sigma=((n+1) s-(s-1)) \sigma$. Let us introduce $m$ as

$$
\begin{aligned}
m & =(n+1) s-(s-1) \\
& =n s+1 .
\end{aligned}
$$

Let us recall the assumption of independent slots, i.e., the state of one slot does not give information about the state of other slots. The probability $\left(\pi_{i}\right)$ that a slot arriving at station $S_{i}$ is full has been approximated in the Appendix. Moreover, there are at most $m$ slots passing by $S_{i}$ during a vacation period, and the time between two consecutive arrivals of a slot at $S_{i}$ that can be used by $S_{i}$ is a multiple of $\sigma$. Therefore, the server vacation period $O_{i}$ has a truncated geometric distribution:

$$
\mathrm{P}\left\{O_{i}=k \sigma\right\}= \begin{cases}\pi_{i}^{k-1},\left(1-\pi_{i}\right) & k=1,2, \cdots m-1 \\ \pi_{i}^{m-1}, & k=m\end{cases}
$$

with $i=0, \cdots, n$.

The first two moments of $O_{i}$ are given by

$$
E O_{i}=\frac{\sigma}{1-\pi_{i}}\left(1-\pi_{i}^{m}\right)
$$

$$
E O_{i}^{2}=\frac{\sigma^{2}}{\left(1-\pi_{i}\right)^{2}}\left\{1+\pi_{i}-(2 m+1) \pi_{i}^{m}+(2 m-1) \pi_{i}^{m+1}\right\}
$$

with $1=0, \cdots, n$

A minipacket service time consists of the duration of a slot rotation in which a minipacket is occupying that slot and the waiting time for a slot that can be used by the station. The 
former time is a constant and equal to $\tau$. For the latter time the distribution (9) is taken. So, a minipacket service time distribution is approximated by

$$
X_{i} \stackrel{D}{=} \tau+O_{i}, \quad i=0, \cdots, n .
$$

where $\stackrel{D}{=}$ denotes equality in the distribution.

The first two moments of $X_{i}$ are given by

$$
\begin{array}{ll}
E X_{i}=s \sigma+E O_{i}, & i=0, \cdots, n \\
E X_{i}^{2}=s^{2} \sigma^{2}+2 s \sigma E O_{i}+E O_{i}^{2} & i=0, \cdots, n .
\end{array}
$$

\section{Message Service Time $Y_{i}$}

Since a message consists of $Z_{i}$ minipackets, the message service time $Y_{i}$ is equal to

$$
Y_{i}=\sum_{j=1}^{Z_{i}} X_{i}^{(j)}
$$

where random variable $X_{i}^{(j)}$ denotes the service time of the $j$ th minipacket within a message arriving at $S_{i}$, with the distribution (12).

Let us now evaluate the first two moments of $Y_{i}$. From (15) we get (see also [18, p. 388]):

$$
\begin{aligned}
E Y_{i} & =\gamma_{i} E X_{i} \\
E Y_{i}^{2} & =\gamma_{i} E X_{i}^{2}+\left(E Z_{i}^{2}-\gamma_{i}\right) \cdot\left(E X_{i}\right)^{2}
\end{aligned}
$$

with $i=0, \cdots, n$.

\section{E. Waiting Time and Delay}

Now the results for the $M|G| 1$ queue with server vacation model and exhaustive service discipline are used to obtain the expected message waiting time. We have from [26] and [28]

$$
E W_{i}=\frac{\gamma_{i} E Y_{i}^{2}}{2\left(1-\lambda_{i} E Y_{i}\right)}+\frac{E O_{i}^{2}}{2 E O_{i}}, \quad i=0, \cdots, n .
$$

The expected message delay consists of the following components: 1) the expected message waiting time $\left.\left(E W_{i}\right), 2\right)$ the expected service time of all but the last minipacket of a message $\left.\left(\left(\gamma_{i}-1\right) E X_{i}\right), 3\right)$ the transmission time of the last minipacket $(\sigma)$, and 4$)$ the expected propagation time of the last minipacket from $S_{i}$ to the destination $\left(\tau_{i}\right)$. So, we have

$$
E T_{i}=E W_{i}+\left(\gamma_{i}-1\right) E X_{i}+\sigma+\tau_{i}, \quad i=0, \cdots, n .
$$

To determine the expected minipacket waiting time $E V_{i}$, i.e., the waiting time of a customer in the $M^{\mathrm{B}}|\mathrm{G}| 1$ model with a server vacation, the following exact formula [19] can be used:

$$
E V_{i}=E W_{i}+\frac{E X_{i}}{2}\left(\frac{E Z_{i}^{2}}{\gamma_{i}}-1\right), \quad i=0, \cdots, n
$$

\section{F. Discussion}

The model provides an implicit estimate of $E O_{i}$ (and thus $E X_{i}$ ) and of $\pi_{i}$. An explicit solution would require solving a system of $n+1$ equations with $n+1$ unknown variables each of order $m$. In a symmetric case this reduces to solving one equation of the same order. So, a numerical solution technique has to be used in the general case to determine values of $\pi_{i}$ and of $E O_{i}$. Let us now summarize the basic modeling assumptions:

- the load from all the stations $S_{j}(j \neq i)$ is represented solely by the load intensity $\lambda_{j} \gamma_{j}$. This way only the first moments of interarrival times and message lengths at $S_{j}(j \neq i)$ are used;

- the protocol overhead due to passing empty slots can be adequately modeled by including it in $\pi_{i}$;

- slots are independent, i.e., it is assumed that the state of one slot does not give any information about the states of the other slots; and

- $O_{i}$ are i.i.d., $X_{i}$ are i.i.d., and $O_{i}$ and $X_{i}$ are mutually independent, and consequently the queue lengths at $S_{i}$ are mutually independent, $i=0, \cdots, n$.

The assumption of slot independence and the approximation of $\pi_{i}$ have lead to the truncated geometric distribution for the server vacation period. Including the slot dependence would lead to a different (and more complex) server vacation distribution. The assumption $s \leq n+1$ has been used for determining the stability conditions and for the recognition of the best and the worst performance pattern if all the queues are unstable. However, it is not an essential one for the delay estimate. It is possible to extend the model for that case as well.

Note that at high loads the variance of the message delay can be large because of the large variance of the duration of the protocol overhead due to passing empty slots in the ring. Note also that if the sufficient stability condition is not satisfied and the necessary one is, the model provides an estimate of the expected delays given that all the queues are stable.

\section{Testing And Analysis of the Model}

The simulation model of CFR against which the analytic model is checked is a detailed one. It is written in SIMULA and is documented in [20] and [30]. The analytic model has been tested by comparing the expected message delays to the results of simulations. $90 \%$ confidence intervals have been obtained. Where that was not possible only a point estimate of the delay (marked by $\Delta$ ) is shown in the figures.

Configurations, system parameters, and workload models expected to be typical for high speed local area networks have been used. We present them as follows: configuration: cable length $=5$ and $1 \mathrm{~km}$, number of stations $=40$ and 10 ; system parameters: transmission rate $=140 \mathrm{Mb} / \mathrm{s}$, slot information field $=512$ bits, overhead in slot $=48$ bits, latency register $=24$ bits; and workload: average message length $=7100$ and 3000 bits, a symmetric and an asymmetric load. In all the examples an equal distance between the neighboring stations has been assumed, i.e., $\tau_{i, i+1}=\tau_{j, j+1}, i, j=0, \cdots, n$. Exponential and bimodal message length distributions have been assumed. 
Note that in the CFR [29] the slot information field length is 256 bits. We have taken another value, 512 bits, in order to be able to do a comparative performance analysis of the selected slotted ring protocols. Such a choice does not change the qualitative behavior of the protocol.

The expected message delay of the CFR access mechanism versus load is depicted in Figs. 7 through 11. An exponential message length distribution has been used except for the case of Fig. 11 where the following bimodal distribution has been taken:

$$
\mathrm{P}\{\text { message length }=x\}= \begin{cases}0.78 & x=512(\mathrm{~b}) \\ \beta: 0.22 & x=30000(\mathrm{~b})\end{cases}
$$

This distribution has been chosen because we wanted to test the model using a distribution with the same expected value of 7100 bits and a second moment about twice as large as in the case of the exponential distribution. Moreover, a bimodal distribution was thought to be a realistic one [31]. Let us now discuss the accuracy of the model.

The $M^{B}|G| 1$ vacation model has the following properties. Its estimates fall within the confidence intervals (halfwidths of which are less than $10 \%$ of the mean obtained by the simulations) of the expected delays for low and moderate symmetric loads until a relative load of about 0.7 .

To highlight the accuracy of the model at high load, a set of experiments has been done which are presented in Table VI. The configuration and the load correspond to the one shown in Fig. 7. One can observe that for loads larger than 0.8 relative load the analytic model overestimates the expected delays compared to the simulation. Due to the large variance of the message delays at high loads no sufficiently accurate simulation results have been obtained for load values larger than $110 \mathrm{Mb} / \mathrm{s}$. At very high loads the model however underestimates the expected delays (see also Table VII, high loads).

An example of an asymmetric load has been chosen with the same configuration and load parameters as in Fig. 7, except for the following. Station $S_{0}$ has $10 \%$ of the total load and all other stations an equal share of the remaining $90 \%$ of the load. The load is balanced, i.e., at each station the intensity of the outgoing traffic equals the intensity of the incoming traffic. In particular, we have the following routing matrix: $p_{i j}=0$, $i=j ; p_{0 j}=1 / 39, j>i ; p_{i j}=p_{i k}=p_{j i}, i \neq j \neq k$, $i, j, k>0$. The results are presented in Table VII. The delays for high load station $S_{0}$ and for one of the low load stations $S_{19}$ are shown. The delay estimates of the analytic model for station $S_{19}$ fall within the confidence interval for all load values. Let us now analyze the delay estimates of the analytic model for station $S_{0}$. They fall within confidence intervals for load values between 50 and $80 \mathrm{Mb} / \mathrm{s}$. Some overestimate appears for loads smaller than $30 \mathrm{Mb} / \mathrm{s}$. However, confidence intervals could not be obtained for load values larger than 85 $\mathrm{Mb} / \mathrm{s}$. However, the delays are much larger in the simulation model than in the analytic model. It should be noted again that in the high load cases especially in Table VII where no confidence intervals could be obtained, the simulation results (as well as the analytic ones) are only indicative. The analytic model thus provides good estimates for the light load stations

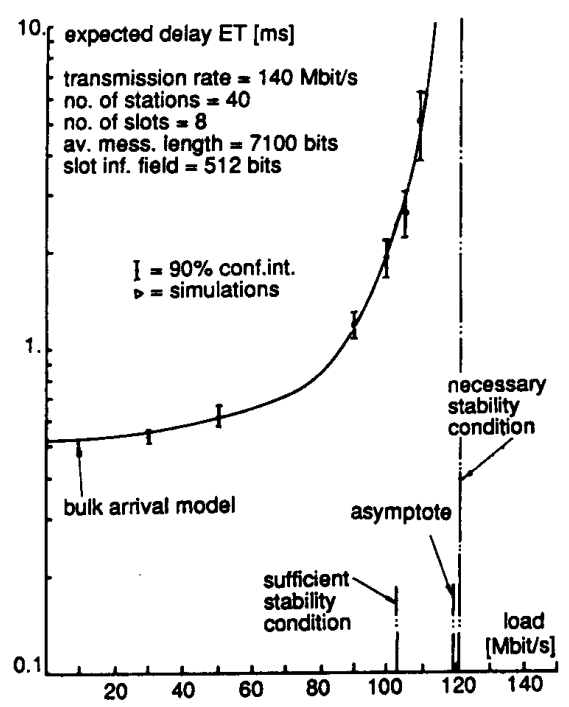

Fig. 7. Message delay versus offered load with 40 stations, 8 slots, and an average message length of 7100 bits.

for all load values and good estimates for the high load station for loads up to the relative system load 0.65 .

The model is more accurate for a larger number of stations (see Figs. 7 and 9). We attribute this to the smaller correlation between activities at $S_{i}$ and other stations in these cases. So, the assumption that $O_{i}$ and $X_{i}$ are i.i.d. is less justifiable in this case.

The model is expected to be more accurate if there are more slots in the ring (see Figs. 9 and 10). The smaller accuracy when there are less slots in the ring is attributed to the assumption of independent slot occupancy, which is more justifiable when there are more slots in the ring. However, this property is not clearly visible in the figures.

The accuracy of the model seems not to depend very much on the average message lengths in the experiments conducted (see Figs. 7 and 8). The model however appears to be slightly more accurate with shorter messages. The accuracy of the model seems also to be very good for both message length distributions (see Figs. 7 and 11).

Note that if $n=0$ and $s=1$ the solution for $E T_{i}$ is exact. It is however, not exact for $s=1$ and $n>0$. An exact solution for that case under a symmetric load is given in [36]. So, the bulk arrival model is very accurate in all the cases studied except at very high loads and for $s=1$.

\section{Performance Evaluation}

The results of the performance evaluation of the CFR access mechanism using our analytic model are shown in Figs. 7 through 17. Figs. 7 through 11 also show simulation results and have already been discussed in the previous section. Figs. 12 through 17 are used to study the sensitivity of the CFR access mechanism with respect to the following parameters: the number of active stations, the transmission rate, the expected message length, and the slot information field length, respectively. All the other parameters are kept 


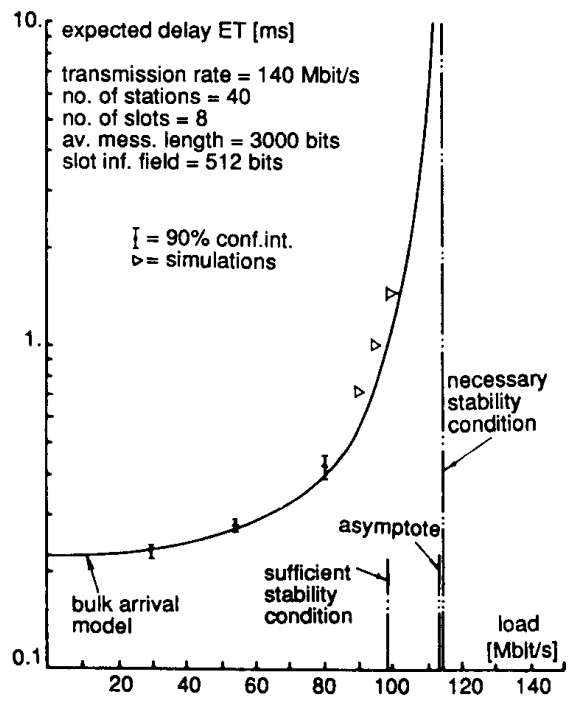

Fig. 8. Message delay versus offered load with 40 stations, 8 slots, and an average message length of 3000 bits.

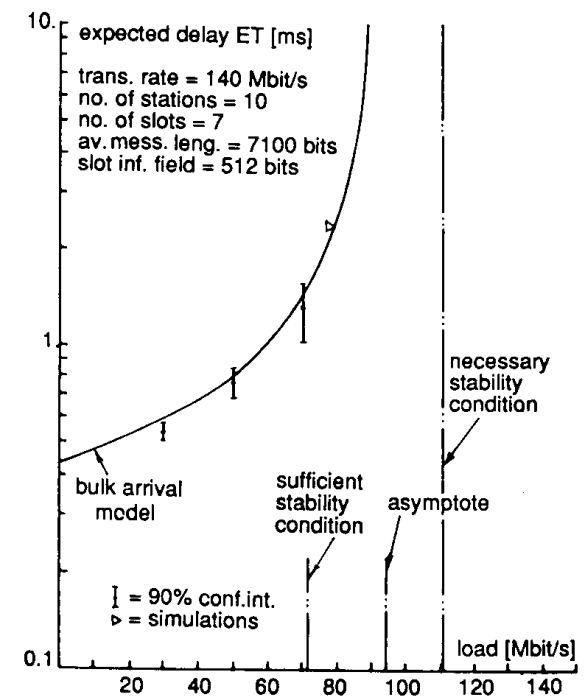

Fig. 9. Message delay versus offered load with 10 stations, 7 slots, and an average message length of 7100 bits.

unchanged and are the same as for Fig. 7. A load intensity of $80 \mathrm{Mb} / \mathrm{s}$ is used except in Fig. 16 where a relative load of 0.65 is used. The same relationship is shown in Fig. 17 as in Fig. 7 except that the slot information field is 256 bits as in the original CFR. In some of the figures the delays of the CFR where the restriction that only one slot at a time can be used by a station is lifted are shown for a comparison (this is the CFRV basic access mechanism). The results for the CFRV have been obtained using the model of [36]. Let us now evaluate the results.

The stability conditions of the CFR access mechanism [see relations (6) and (7)] show that the maximum carried load

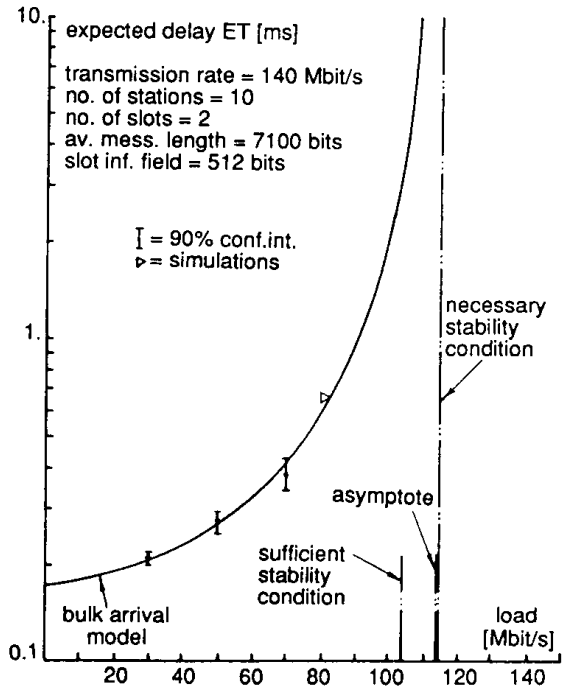

Fig. 10. Message delay versus offered load with 10 stations, 2 slots, and an average message length of 7100 bits.

depends on the number of stations, the slot duration, and the first moments of message interarrival times and bulk size. The sufficient condition also depends on the number of slots. The necessary condition does not depend on the number of slots. Hence, it also does not depend on the ring latency.

The lower bound on the maximum carried load is given by the sufficient stability condition and the upper bound on the maximum carried load by the necessary stability condition (see Figs. 7 through 11). So, a realization of the CFR would guarantee the minimum carried load, but would, depending on stochastic behavior, support loads up to the maximum carried load. In the experiments shown in the figures, the CFR access mechanism proves to be capable of carrying loads that are not far off from its transmission rate, e.g., about $110 \mathrm{Mb} / \mathrm{s}$ in the case of Fig. 7 (also Table VI).

The CFR access mechanism shows a steep increase of the delays when the load is at about 0.7 of the maximum carried load estimated by the model (see Figs. 7 through 11 and Tables VI and VII). This indicates that this access mechanism is very sensitive to sudden changes of the load in that region of the load values. So, a sudden and unexpected increase of the delays may be observed by a user. Some other slotted ring protocols in particular Orwell do not have this effect so strongly (see [35]).

The expected delay is quite sensitive to the number of slots in the ring (see Figs. 9, 10, and 12 and Table I). It performs much better if the number of slots is very small (e.g., 2) than if it is large (e.g., 40), see Fig. 12. This is the opposite of, e.g., Orwell where the performance is better with a larger number of slots, e.g., 7, than with a smaller one, e.g., 2 (see [35]). The main reason is the fact that only one slot at a time can be occupied by a station. This property has a larger influence on the performance if the number of slots is larger (i.e., the ratio between the number of active stations and the number of slots decreases). In that case the delays are larger. 


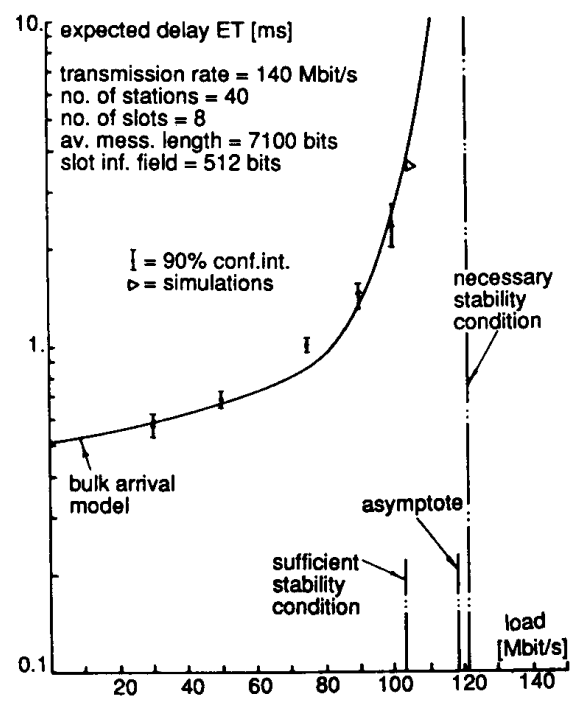

Fig. 11. Message delay versus offered load with 40 stations, 8 slots, an average message length of 7100 bits, and bimodal message length distribution.

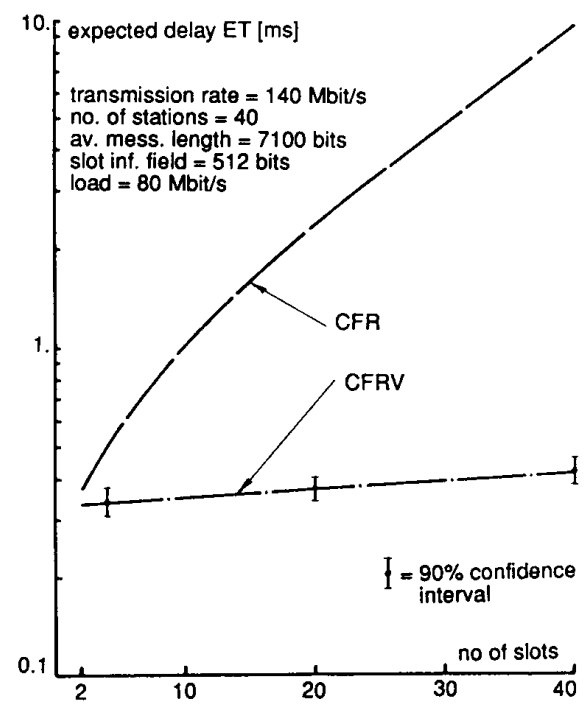

Fig. 12. Message delay versus number of slots.

TABLE I

The Cable Length Versus the Number of Slots

WHEN $w=140 \mathrm{Mb} / \mathrm{s}, n+1=40$, AND $n=512 \mathrm{~b}$

\begin{tabular}{lccccc}
\hline no. of slots & 2 & 10 & 20 & 30 & 40 \\
cable length $(\mathrm{km})$ & 0 & 6.63 & 14.63 & 22.63 & 30.63 \\
\hline
\end{tabular}

The expected delays depend on the number of stations in the ring (see Figs. 7, 9, and 13). The delays decrease asymptotically with the increase of the number of active stations. The performance differs significantly for the smaller and medium numbers of active stations (e.g., between 8 and 60 in Fig. 13). As already mentioned if $n+1<s$ a part of the ring capacity is wasted since at most $n+1$ slots can be

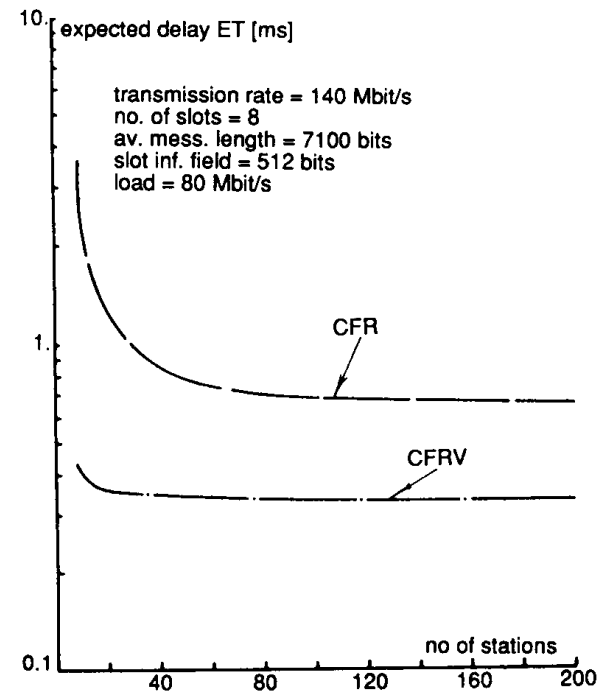

Fig. 13. Message delay versus number of stations.

occupied at a time. There are two main reasons for the increase of the delays when $n+1$ gets close to $s$. The first one is the fact that only one slot at a time can be occupied by a station. This effect has already been explained. The second reason is queueing at $S_{i}$ which takes a larger share in the total delay than waiting for the access to the medium. Because of the constant total load queueing at $S_{i}$ is larger if the number of active stations is smaller. The latter effect can also be observed in other slotted ring protocols, see [36] and [35].

The message delay versus transmission rate is shown in Fig. 14 (see also Table II which shows the number of slots in the ring versus the transmission rate for the case of Fig. 14). The relative load is held constant at 0.65 , e.g., $80 \mathrm{Mb} / \mathrm{s}$ load at $140 \mathrm{Mb} / \mathrm{s}$ transmission rate. Above $300 \mathrm{Mb} / \mathrm{s}$ the CFR has increasing delays, mainly because of the increase of the number of slots. This is opposed to the CFRV indicating that the use of up to one slot at a time by a station is a limitation in the implementation of the CFR at very large transmission rates, e.g., up to $1 \mathrm{~Gb} / \mathrm{s}$.

The expected message delay varies with respect to the average message length (see Figs. 7, 8, and 15). The change in message length causes a change in the expected number of minipackets in a message. This also causes a change in the expected number of minipackets which are the last ones in messages and which are therefore only partially filled in by data. Table III shows the relative load $\rho$ versus the expected message length [see relation (1)]. Therefore, when the average message length changes, the relative load on the network also changes. The performance strongly degrades when the expected message length is smaller than the slot information field length due to the large relative load caused by lots of slots which are only partially filled by useful data (this explains the delay decline with increasing message length for short messages). Let us now analyze the case where the expected message length is larger than the slot information field. Note that when the expected message length increases given the 


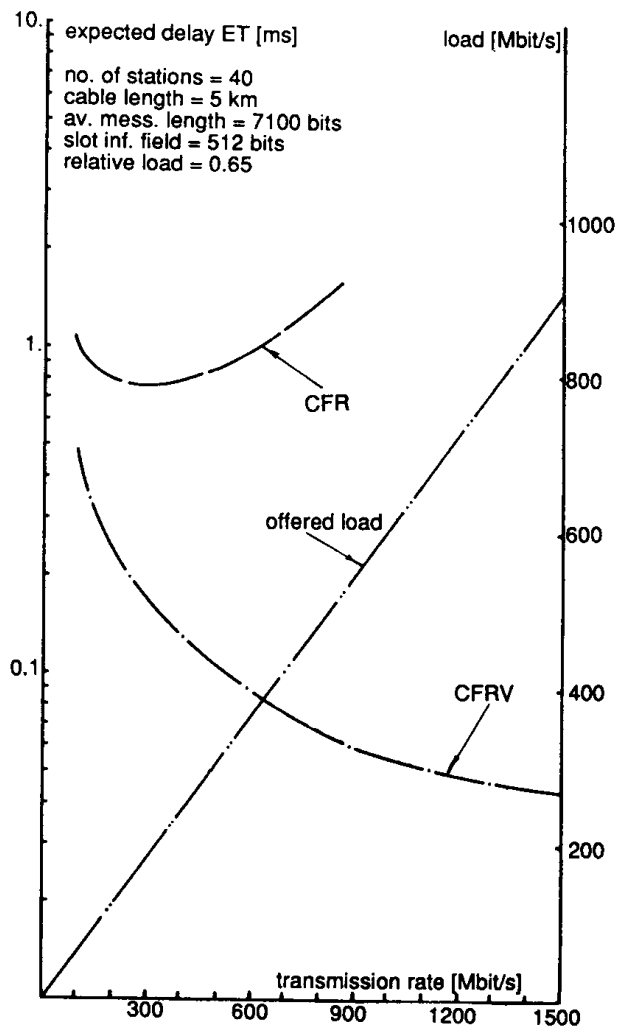

Fig. 14. Message delay and offered load versus transmission rate.

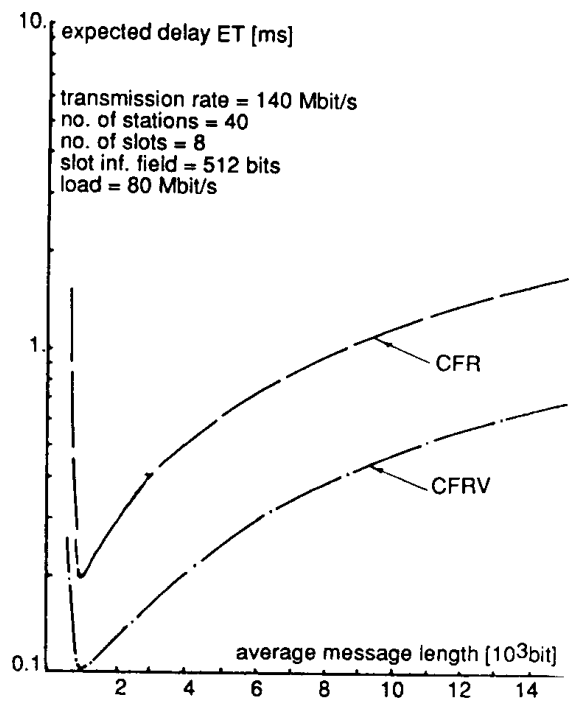

Fig. 15. Message delay versus average message length.

total load on the system, the load on the network decreases. However, the larger the expected message length the larger the message delay. The increase of the delays in Fig. 15 is mainly due to the fact that only one slot at a time can be used by a station.
TABLE II

The Number of Slots Versus Transmission Rate When $n+1=40$, CABLE LENGTH $=5 \mathrm{~km}$, AND $\nu=512 \mathrm{~b}$

\begin{tabular}{l|c|c|c|c|c}
\hline transmission rate $(\mathrm{Mb} / \mathrm{s})$ & 100 & 140 & 565 & 800 & 848 \\
no. of slots & 7 & 8 & 27 & 38 & 40 \\
\hline
\end{tabular}

TABLE III

The Relative Load $\rho$ Versus the Expected Message Length WHEN $w=140 \mathrm{Mb} / \mathrm{s}$, TOTAL LOAD $=80 \mathrm{Mb} / \mathrm{s}$, AND $\nu=512 \mathrm{~b}$

\begin{tabular}{l|c|c|c|c|c|c}
\hline $\begin{array}{l}\text { exp. message length } \\
\left(10^{3} \text { b) }\right.\end{array}$ & 0.5 & 2 & 7.1 & 14 & 28 & 56 \\
relative load & 1.00 & 0.71 & 0.65 & 0.64 & 0.63 & 0.63 \\
\hline
\end{tabular}

The sensitivity of the CFR access mechanism with respect to the slot information field length is depicted in Fig. 16 for load values of 60,80 , and $100 \mathrm{Mb} / \mathrm{s}$ (see also Fig. 17). The delay function is discontinuous because of the change in the number of slots in the ring with the increase of the information field length (see Table IV). The relative load $\rho$ is shown in Table $\mathrm{V}$. This access mechanism performs best with an information field length between 1024 and 2048 bits. This is because of the change in relative load $\rho$ and the number of slots in the ring. If the information field length decreases the relative load increases since the overhead gets large in relation to the slot length. If the information field increases the relative load also increases. This happens because of the decrease of the expected number of minipackets in a message which causes the same effect as already explained in the case of a change in the expected message length. The sharp increase in delays in Fig. 16 can be compared to the increase in delays when the offered load is changed (e.g., in Figs. 7 through 11) which happens if the relative load is larger than about 0.7 . This effect is however combined with the effect of changing the number of slots. If the slot information field is between 1024 and 2048 bits the relative load is small and the number of slots as well, so the performance of the CFR is at its best. Note that the choice of the information field length in practice is to a large extent determined by the message length distribution. Namely, if the most dominant traffic class has a constant message length (e.g., voice) the best performance of the protocol could be expected if the message fits into an integer number of slots. The slot information field length should be chosen such that on the one hand, the ratio between the relative load and the offered load is small, and on the other hand the number of slots in a typical application is small. For the workload used here, the CFR access mechanism with 256 bits information field length as proposed in [29] has much worse performance than with 512 bits (see Figs. 7 and 17). Fig. 16 shows that information field lengths between 512 bits and 3072 bits provide good performance. However, when the information field length is in this range the delays for eventual message transmission may become unacceptable. A uniframe scheme over the CFR protocol would improve the performance for voice in this case. However, the study in [38] shows that in that case the delays for long messages belonging to the asynchronous traffic class become large. 
TABLE IV

The Number of Slots Versus the Slot Information Field Length When $w=140 \mathrm{Mb} / \mathrm{s}, n+1=40$, and Cable Length $=5 \mathrm{~km}$

\begin{tabular}{c|c|c|c|c|c|c|c|c}
\hline inf. field length (b) & 64 & 128 & 256 & 512 & 1024 & 2048 & 4096 & 8192 \\
no. of slots & 33 & 32 & 16 & 8 & 5 & 3 & 2 & 1 \\
\hline
\end{tabular}

TABLE V

The Relative Load $\rho$ For a $80 \mathrm{Mb} / \mathrm{s}$ LoAd Versus the Slot Information Field Length When $w=140$ Mb/s and ExPECted Message Length $=7100 \mathrm{~b}$

\begin{tabular}{l|c|c|c|c|c|c|c|c|c|}
\hline inf. field length (b) & 64 & 128 & 256 & 512 & 1024 & 2048 & 4096 & 8192 \\
relative load & 1.01 & 0.73 & 0.69 & 0.65 & 0.64 & 0.67 & 0.76 & 0.97 \\
\hline
\end{tabular}

TABLE VI

Message Delay Versus Offered Load for a High Load Case

\begin{tabular}{|c|l|l|l|l|l|l|}
\hline \multirow{2}{*}{$\begin{array}{l}\text { offered } \\
\text { load } \\
\text { [Mbit/s] }\end{array}$} & an.model & \multicolumn{2}{|c|}{ simulation } & an.model & \multicolumn{2}{|c|}{ simulation } \\
\cline { 2 - 7 } & $\begin{array}{l}\text { relative } \\
\text { load } \\
\rho\end{array}$ & $\begin{array}{l}\text { carried } \\
\text { load } \\
\text { [Mbit/s] }\end{array}$ & $\begin{array}{l}\text { slot } \\
\text { utiliz }\end{array}$ & $\begin{array}{l}\text { expected } \\
\text { delay } \\
\text { [ms] }\end{array}$ & $\begin{array}{l}\text { expected } \\
\text { delay } \\
\text { [ms] }\end{array}$ & $\begin{array}{l}90 \text { \& } \\
\text { conf.int }\end{array}$ \\
\hline 90 & 0.73 & 90.8 & 0.74 & 1.11 & 1.18 & 0.09 \\
95 & 0.77 & 95.7 & 0.78 & 1.33 & 1.46 & 0.19 \\
100 & 0.81 & 100.8 & 0.82 & 1.83 & 1.83 & 0.22 \\
102 & 0.83 & 102.7 & 0.83 & 2.18 & 2.06 & 0.26 \\
105 & 0.85 & 105.4 & 0.85 & 2.93 & 2.48 & 0.36 \\
110 & 0.89 & 110.2 & 0.89 & 5.29 & 4.75 & 1.12 \\
& & & & & & \\
\hline
\end{tabular}

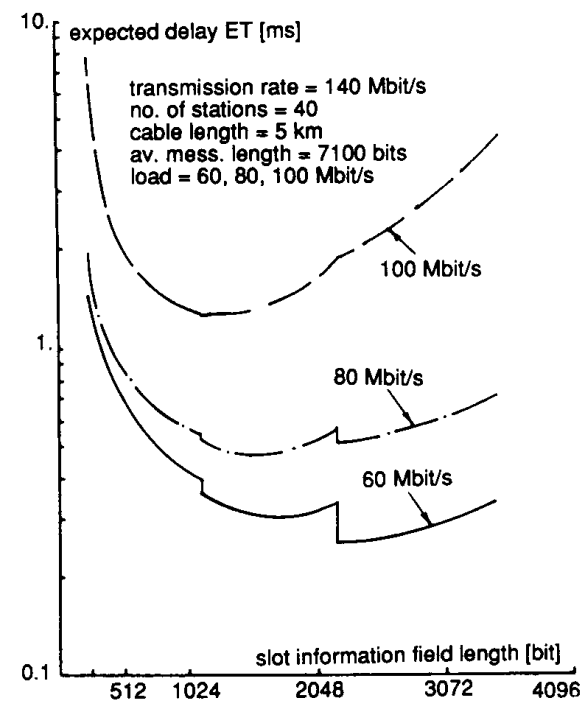

Fig. 16. Message delay versus slot information field length.

Applications: Because of the properties of the CFR access mechanism, we can conclude that it can perform well when the number of slots is small or moderate relative to the number of active stations. This means that in a backbone application with a small number of stations and a large cable length with a large number of slots, this protocol performs worse than in, e.g., an application as a multicomputer or packet switch interconnection structure where the number of slots is small relative to the number of active stations. The CFR access mechanism could also perform well as an integrated

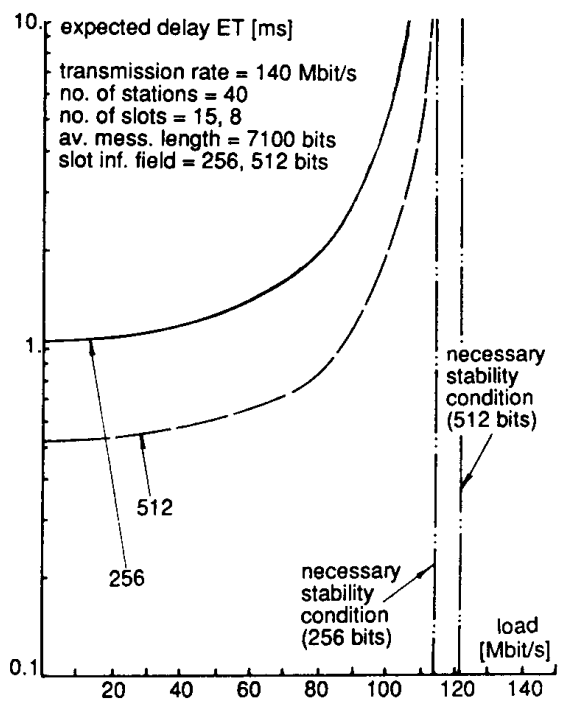

Fig. 17. Message delay versus offered load with 40 stations, an average message length of 7100 bits, and a slot information field length of 256 or 512 bits.

services network, provided that the number of stations is large enough relative to the number of slots which is in general to be expected. However, fulfillment of the quality of service requirements for the different traffic classes, e.g., synchronous and asynchronous traffic is a subject of the study in [38].

\section{CONCLUSION}

The CFR access mechanism has been analyzed. It has been shown that CFR can be represented by a multiqueue multiple 
TABLE VII

Message Delay Versus Offered load for an asymmetric load Case

\begin{tabular}{|c|c|c|c|c|c|c|c|c|c|c|c|}
\hline \multirow{3}{*}{$\begin{array}{l}\text { offered } \\
\text { load } \\
{[\mathrm{Mbit} / \mathrm{s}]}\end{array}$} & \multirow{3}{*}{$\begin{array}{l}\text { an.model } \\
\text { relative } \\
\text { load } \\
\rho\end{array}$} & \multirow{2}{*}{\multicolumn{2}{|c|}{ simulation }} & \multicolumn{4}{|c|}{ station $\mathrm{s}_{0}$} & \multicolumn{4}{|c|}{ station $\mathrm{S}_{19}$} \\
\hline & & & & \multirow{2}{*}{$\begin{array}{l}\text { an.model } \\
\text { expected } \\
\text { delay } \\
\text { [ms ] }\end{array}$} & \multicolumn{3}{|c|}{ simulation } & \multirow{2}{*}{$\begin{array}{l}\text { an.model } \\
\text { expected } \\
\text { delay } \\
\text { [ms] }\end{array}$} & \multicolumn{3}{|c|}{ simulation } \\
\hline & & $\begin{array}{l}\text { carried } \\
\text { load } \\
{[\text { Mbit } / \mathrm{s}]}\end{array}$ & $\begin{array}{l}\text { slot } \\
\text { utiliz. }\end{array}$ & & $\begin{array}{l}\text { carried } \\
\text { load } \\
{[\mathrm{Mbit} / \mathrm{s}]}\end{array}$ & $\begin{array}{l}\text { expected } \\
\text { delay } \\
{[\mathrm{ms}]}\end{array}$ & $\begin{array}{l}958 \\
\text { conf. } \\
\text { int. }\end{array}$ & & $\begin{array}{l}\text { carried } \\
\text { load } \\
{[\text { Mbit/s] }}\end{array}$ & $\begin{array}{l}\text { expected } \\
\text { delay } \\
{[\mathrm{ms}]}\end{array}$ & $\begin{array}{l}958 \\
\text { conf. } \\
\text { int. }\end{array}$ \\
\hline 10 & 0.08 & 10.0 & 0.08 & 0.69 & 1.1 & 0.55 & 0.08 & 0.54 & 0.31 & 0.56 & 0.18 \\
\hline 30 & 0.24 & 30.2 & 0.24 & 0.81 & 3.3 & 0.71 & 0.08 & 0.57 & 0.84 & 0.53 & 0.10 \\
\hline 50 & 0.41 & 50.1 & 0.41 & 1.02 & 5.1 & 0.91 & 0.11 & 0.62 & 1.32 & 0.62 & 0.10 \\
\hline 70 & 0.57 & 69.9 & 0.56 & 1.51 & 7.0 & 1.67 & 0.48 & 0.71 & 1.79 & 0.78 & 0.17 \\
\hline 80 & 0.65 & 78.9 & 0.64 & 2.37 & 7.8 & 3.10 & 1.49 & 0.84 & 2.33 & 0.89 & 0.13 \\
\hline 85 & 0.69 & 83.6 & 0.68 & 3.95 & 8.3 & 5.50 & - & 0.98 & 2.14 & 0.99 & 0.15 \\
\hline 90 & 0.73 & 88.2 & 0.72 & 9.14 & 8.6 & 24.81 & - & 1.11 & 2.25 & 1.12 & 0.22 \\
\hline 93 & 0.75 & 90.0 & 0.73 & 33.69 & 8.9 & 40.27 & - & 1.20 & 2.31 & 1.16 & 0.19 \\
\hline
\end{tabular}

cyclic server model with a 1-limited service discipline and with a restriction that only one server at a time can be serving a queue. A new approximate analytic model which is a $M|G| 1$ vacation model with a bulk Poisson arrival process of minipackets has been developed and a performance evaluation has been done. The conclusions concerning the analytic model can be summarized as follows:

- the exact necessary stability condition and the exact sufficient stability condition for the CFR access mechanism have been stated starting from multiple cyclic server models of the access mechanism;

- the $\mathrm{M}^{\mathrm{B}}|\mathrm{G}| 1$ vacation model provides a good estimate of the expected message delay over a wide range of parameters under a symmetric load and symmetric traffic pattern for $s>1$; the estimates deviate up to $10 \%$ over a range of relative load going from 0 to 0.7 from the point estimates obtained by simulations; the model gives a good qualitative insight into the delays, and at lower and medium loads an accurate delay estimate as well;

- for high loads the model underestimates the expected message delay; an analysis of a high load case shows a good correspondence of the simulation and analytic results;

- the $M^{\mathrm{B}}|\mathrm{G}| 1$ vacation model is expected to be less accurate for asymmetric loads; it provides good approximations of the expected message delays under asymmetric load in the case studied (one station producing $10 \%$ of the load); the approximation for low load stations falls within the $90 \%$ confidence interval and deviates at most $10 \%$ from the point estimates obtained by the simulation; the estimate for the high load station is good up to 0.65 relative system load and produces an underestimate for higher relative loads; and

- the model is more accurate with more active stations in the ring.

The conclusions concerning the performance of the CFR access mechanism can be summarized as follows.

- the maximum carried load is limited by its lower and upper bounds, determined by the stability conditions;
- the CFR access mechanism performs better if the ratio between the number of slots and the number of active stations is small;

- the performance of the CFR access mechanism is better with smaller expected message lengths provided they are larger than the slot length;

- the CFR access mechanism shows good performance at higher transmission rates only if the number of slots is small relative to the number of active stations;

- the following two parameters have the strongest influence on the performance of the CFR if the system parameter slot information field length is changed: the relative load, and the number of slots, the relative load being related to the number of slots; the smaller it is the better the performance is; a slot information field length between 512 bits and 3072 bits provides good performance for representative system parameters and workloads; and

- the performance of the CFR access mechanism makes it interesting for applications where the ratio between the number of active stations and the number of slots is large or moderate, e.g., as a multicomputer interconnection structure.

This model has been used in [38] and [24] for a comparative analysis of slotted- and token ring protocols at high transmission rates.

\section{APPENDIX \\ PROBABILITY $\pi_{i}$}

In order to estimate $\pi_{i}$ we proceed in three steps. At first we analyze the slot occupancy which is directly related to the use of the system capacity. The probability $\pi_{i}$ is approximated next, by introducing a probability $q_{i}$. Finally $q_{i}$ is heuristically approximated. In this section we assume that the number of active stations is larger than one, i.e., $n>0$, except when determining $\pi_{i}$ in formula (A.3).

\section{A. Slot Occupancy}

Each minipacket occupies a slot for a time $\tau$. However, the system capacity is used somewhat longer per minipacket 
transfer since there is some overhead due to the protocol properties. Let $\alpha_{i}$ denote the expected duration of the slot occupancy per minipacket transfer including this overhead for minipackets which are originated by $S_{i}$.

Extra capacity is wasted after a slot carrying a minipacket (which is originated by $S_{i}$ ) is released (by $S_{i}$ ) because of the following:

1) passing the slot empty to the first downstream station $S_{i+1}$, which on the average takes $\tau /(n+1)$ time units, and

2) furthermore, passing the slot around empty among stations because of one of the two following reasons:

- the queue at a visited station $S_{k}(k \neq i)$ is empty and there is a nonempty station $S_{j}(j \neq i)$ which can use an empty slot, or

- the queue at a visited station $S_{k}(k \neq i)$ is nonempty and the station has a full slot in the ring.

Related to overhead 2), let us define $q_{i}$ as follows: for any station $S_{k}(k \neq i)$

$q_{i}=\mathrm{P}\left\{\right.$ either $S_{k}$ is empty and there is a nonempty station $S_{j}(j \neq i, j \neq k)$ that has no full slot in the ring, or $S_{k}$ is nonempty and has a full slot in the ring $\mid$ an empty slot arrives at $\left.S_{k}\right\}$.

Assuming the independence of slots, overhead 2) can be determined by noting that upon arrival of a slot at station $S_{j}$ $(j \neq i)$ either the slot is passed with probability $q_{i}$ to the downstream station, with mean switchover time $\tau /(n+1)$, or the overhead period of type 2 ) is ended with probability $1-q_{i}$.

$\alpha_{i}$ can now be determined as the sum of the minipacket transfer time, the duration of overhead 1), and the expected duration of overhead 2) as follows.

$$
\begin{aligned}
\alpha_{i} & =\tau+\frac{\tau}{n+1}+\frac{\tau}{n+1} \sum_{j=0}^{\infty} j q_{i}^{j}\left(1-q_{i}\right) \\
& =\tau+\frac{1}{1-q_{i}} \cdot \frac{\tau}{n+1}, \quad i=0, \cdots, n, n>0 .
\end{aligned}
$$

\section{B. Approximation of $\pi_{i}$}

We have argued that the system capacity is used for a duration $\alpha_{i}$ per minipacket transfer. Since $\pi_{i}$ is strongly related to the slot occupancy, as observed by $S_{i}$, we approximate $\pi_{i}$ as follows:

$$
\pi_{i}=\frac{1}{s} \sum_{j=0, j \neq i}^{n} \lambda_{j} \gamma_{j} \alpha_{i} \quad i-0, \cdots, n, n>0
$$

For $n=0$, we define $\pi_{i}=0, i=n$. Including this in (A.2) and using (A.1) we get

$$
\pi_{i}= \begin{cases}\sum_{j=0, j \neq i}^{n} \lambda_{i} \gamma_{i} \sigma\left(1+\frac{1}{n+1} \cdot \frac{1}{1-q_{i}}\right), & i=0, \cdots, n, n>0 \\ 0, & i=n, n=0 .\end{cases}
$$

\section{Approximation of $q_{i}$}

Since the two events in the definition of $q_{i}$ are mutually exclusive, we have

$$
q_{i}=q_{i}^{(1)}+q_{i}^{(2)}
$$

with

$q_{i}^{(1)}=\mathrm{P}\left\{\right.$ station $S_{k}$ is empty and there is a nonempty station $S_{j}(j \neq i, j \neq k)$ that has no full slot in the ring | an empty slot arrives at $\left.S_{k}\right\}$,

and

$q_{i}^{(2)}=\mathrm{P}\left\{\right.$ station $S_{k}$ is nonempty and has a full slot in the ring $\mid$ an empty slot arrives at $\left.S_{k}\right\}$ for any station $S_{k}, k \neq i$.

Let us now further approximate heuristically as an average over all stations excluding $S_{i}$ :

$$
\begin{aligned}
q_{i}^{(1)}=\frac{1}{n} \sum_{k=0, k \neq i}^{n} \mathrm{P}\{ & \text { station } S_{k} \text { is empty and there is a } \\
& \text { nonempty station } S_{j} \text { that has no full } \\
& \text { slot in the ring, }(j \neq i, j \neq k) \mid \text { an } \\
& \text { empty slot arrives at } \left.S_{k}\right\}
\end{aligned}
$$

which leads to

$$
\begin{aligned}
& q_{i}^{(1)}=\frac{1}{n} \sum_{k=0, k \neq i}^{n} \mathrm{P}\left\{S_{k} \text { is empty }\right\} \cdot\left(1-\prod_{j=0, j \neq i, j \neq k}^{n} \mathrm{P}\left\{S_{j}\right. \text { is }\right. \\
&\text { empty or is nonempty and has }\} \\
&\text { a full slot }\}))
\end{aligned}
$$

$\mathrm{P}\left\{S_{k}\right.$ is nonempty $\}$ is approximated by the product of the minipacket arrival rate and minipacket service time, i.e., $\lambda_{k} \gamma_{k} E X_{k}$. The probability $\mathrm{P}\left\{S_{j}\right.$ is nonempty and has a full slot in the ring $\}$ is approximated by $\mathrm{P}\left\{S_{j}\right.$ is nonempty $\} \cdot \mathrm{P}\left\{S_{j}\right.$ has a full slot in the ring $\}$. $\mathrm{P}\left\{S_{j}\right.$ has a full slot in the ring $\}$ is approximated by the ratio between the slot occupation time and the minipacket service time, i.e., $\tau / E X_{j}$. This yields

$$
\begin{aligned}
q_{i}^{(1)}=\frac{1}{n} & \sum_{k=0, k \neq i}^{n}\left\{\left(1-\lambda_{k} \gamma_{k} E X_{k}\right) \cdot(1-\right. \\
& \left.\prod_{j=0, j \neq i, j \neq k}^{n}\left(1-\lambda_{j} \gamma_{j} E X_{j}+\lambda_{j} \gamma_{j} E X_{j} \cdot \frac{\tau}{E X_{j}}\right)\right\}
\end{aligned}
$$

From relation (A.5) after some manipulation and using relations (13) and (3), we get

$$
\begin{aligned}
q_{i}^{(1)}= & 1-\frac{1}{n} \sum_{k=0, k \neq i}^{n} \lambda_{k} \gamma_{k} E X_{k}-\prod_{j=0, j \neq i}^{n}\left(1-\lambda_{j} \gamma_{j} E O_{j}\right) \\
& +\frac{1}{n} \sum_{k=0, k \neq i}^{n}\left(\lambda_{k} \gamma_{k} \tau \prod_{j=0, j \neq k}^{n}\left(1-\lambda_{j} \gamma_{j} E O_{j}\right)\right) .
\end{aligned}
$$

Note that if $n=1$, i.e., there are two stations in the ring, expression (A.6) becomes 0 . 
Let us now evaluate $q_{i}^{(2)}$. In Section VI we argued that a number of stable patterns of operation of the ring may exist when all the queues are unstable. In the state that has the best performance, $q_{i}^{(2)}$ is zero (note that it is assumed that $s \leq n+1$ ). If all the queues are stable, the system can stay in one pattern at very high loads for a long time relative to the duration of a message transmission. Since the performance differs significantly in the different patterns, this has a large influence on the variance and on the expected value of the time a message spends in the system and it may eventually lead to instability of the queues. However, this effect is incorporated only by the expected value of the number of stations which are visited by an empty slot and are not served because they have a full slot in the ring. We approximate $q_{i}^{(2)}$ heuristically averaging over all stations, excluding $S_{i}$ by

$$
q_{i}^{(2)}=\frac{1}{n} \sum_{k=0, k \neq i}^{n} \mathrm{P}\left\{\text { station } S_{k}\right. \text { is nonempty and }
$$

has a full slot in the ring, $(j \neq i, j \neq k)$ | an empty slot arrives at $S_{k}$ \}.

Further, we have

$$
\begin{aligned}
q_{i}^{(2)}=\frac{1}{n} \sum_{k=0, k \neq i}^{n} \mathrm{P}\{ & \text { station } S_{k} \text { has a full slot in the } \\
& \text { ring that is not the last of the bulk }\} \\
& +\mathrm{P}\left\{\text { station } S_{k}\right. \text { has a full slot in the } \\
& \text { ring which is the last of the bulk }\} \\
& \left.\mathrm{P}\left\{\text { station } S_{k} \text { is nonempty }\right\}\right) .
\end{aligned}
$$

By approximating $\mathrm{P}\left\{S_{k}\right.$ is nonempty $\}$ by $\lambda_{k} \gamma_{k} E X_{k}, k \neq i$, we obtain

$$
\begin{aligned}
q_{i}^{(2)} & =\frac{1}{n} \sum_{k=0, k \neq i}^{n}\left(\left(\lambda_{k}\left(\gamma_{k}-1\right) \tau\right)+\lambda_{k} \tau \cdot \lambda_{k} \gamma_{k} E X_{k}\right) \\
& =\frac{1}{n} \sum_{k=0, k \neq i}^{n} \lambda_{k} \gamma_{k} \tau-\frac{1}{n} \sum_{k=0, k \neq i}^{n} \lambda_{k} \tau\left(1-\lambda_{k} \gamma_{k} E X_{k}\right)
\end{aligned}
$$

From (A.4), (A.6), and (A.7), we finally have that

$$
\begin{aligned}
& q_{i}=1- \frac{1}{n} \sum_{k=0, k \neq i}^{n}\left(\lambda_{k}\left(1-\gamma_{k}\right) \tau+\left(1-\lambda_{k} \tau\right) \lambda_{k} \gamma_{k} E X_{k}\right) \\
&-\prod_{j=0, j \neq i}^{n}\left(1-\lambda_{j} \gamma_{j} E O_{j}\right) \\
&+\frac{1}{n} \sum_{k=0, k \neq i}^{n}\left(\lambda_{k} \gamma_{k} \tau \prod_{j=0, j \neq k}^{n}\left(1-\lambda_{j} \gamma_{j} E O_{j}\right)\right) \\
& i=0, \cdots, n, n>0 .(\text { A.8 })
\end{aligned}
$$

\section{REFERENCES}

[1] B. van Arem, "Queueing models for slotted transmission systems," Ph.D. dissertation, ISBN 90-9 003 421-8, Dep. Appl. Math., Univ. Twente, Enschede, The Netherlands, June 1990.
[2] Y. Baba, "On the MX/G/1 queue with vacation time," Oper. Res. Lett. vol. 5, no. 2, Elsevier Sc.Pb.B.V., pp. 93-98, July 1986.

[3] British Telecom, "Stable cycles and analytic results for slotted rings," British Telecom Teletraffic Memorandum 84/08, British Telecom Technical Publications, Mar. 1984.

[4] British Telecom, "Stable cycles and analytic results for the Cambridge ring: Further results," British Telecom Teletraffic Memorandum 84/58, British Telecom Technical Publications, Dec. 1984.

[5] W. Bux, "Local area subnetworks: A performance comparison," IEEE Trans. Commun., vol. COM-29, no. 10, pp. 1465-1473, Oct. 1981

[6] L. M. Casey, R. C. Dittburner, and R. C. Gamage, "FXNET: A backbone ring for voice and data," IEEE Commun. Mag., vol. 24, no. 12, pp. 23-28, Dec. 1986

[7] R. B. Cooper and G. Murray, "Queues served in cyclic order," Bell Syst. Tech. J., vol. 48, no. 3, pp. 675-689, Mar. 1969.

[8] J. Chauhan, T. J. King, and A. C. Micallef, "Specification of the Orwell protocol," British Telecom Technical Publications, Issue B.2 (12/89), London, Dec. 1989.

[9] R. M. Falconer, J. L. Adams, and G. M. Walley, "A simulation study of the Cambridge ring with voice traffic," British Telecom Tech. J., vol. 3, no. 2, pp. 85-91, Apr. 1985.

[10] S. W. Fuhrmann and Y. T. Wang, "Mean waiting time approximations of cyclic service systems with limited service," in Proc. Performance '87, P.-J. Courtois and G. Latouche, Eds., North Holland, 1988, pp. $253-265$.

[11] W. K. Giloi, P. Behr, and G. Zuber, "Upperbus, A high speed backbone for metropolitan area networks," in Proc. EFOC/LAN'86, June 1986, pp. $286-289$.

[12] D. Greaves, D. Lioupis, and A. Hopper, "The Cambridge backbone ring," in Proc. IEEE INFOCOM'90, San Francisco, CA, June 1990, pp. $8-14$.

[13] W. P. Groenendijk, "Conservation laws in polling systems," Ph.D. dissertation, Rijksuniversiteit Utrecht, Utrecht, The Netherlands, Jan. 1990.

[14] J. F. Hayes and D. N. Sherman, "Traffic analysis of a ring switched data transmission," Bell Syst. Tech. J., vol. 50, no. 9, pp. 2947-2978, Nov. 1971

[15] A. Hopper and R. M. Needham, "The Cambridge fast ring networking system," IEEE Trans. Comput., vol. 37, no. 10, pp. 1214-1223, Oct. 1988.

[16] A. Hopper, "The Cambridge ring-A local network," in Advanced Techniques for Microprocessor Systems, F. K. Hanna, Ed. Stevenage, U. K.: Peter Pergrinus Ltd., 1980, pp. 67-71,

[17] P. J. B. King and I. Mitrani, "Modeling a slotted ring local area network," IEEE Trans. Comput., vol. C-36, no. 5, pp. 554-561, May 1987.

[18] L. Kleinrock, Queueing Systems, Vol. I. New York: Wiley, 1979.

[19] P. J. Kuehn, "Multiqeueue systems with nonexhaustive cyclic service," Bell Syst. Tech. J., vol. 58, no. 3, pp. 671-698, Mar. 1979.

[20] K. van der Lande, "Design and implementation of a Cambridge fast ring variant simulation model," Rep. 080-87-06, Univ. Twente, The Netherlands, Feb. 1987

[21] W. M. Loucks, V. C. Hamacher, B. R. Preiss, and L. Wong, "Short packet transfer performance in local area ring networks," IEEE Trans. Comput, vol. C-34, no. 11, pp. 1006-1014, Nov. 1985.

[22] I. Mitrani, J. L. Adams, and R. M. Falconer, "A modeling study of the Orwell ring protocol," in Teletrafic Analysis and Computer Performance Evaluation, O. Boxma, J. Cohen, and H. Tijms, Eds. Amsterdam, The Netherlands: Elseviers Sc.Pb. B. V., 1986, pp. 429-438.

[23] R. J. I. Morris and Y. I. Wang, "Some results for multi-queue systems with multiple cyclic servers," in Performance of Computer Communication Systems, H. Rudin and W. Bux, Eds. Amsterdam, The Netherlands: Elsevier Sc.Pb. B. V. 1984, pp. 4.2A-5-1-4.2A-5-7,

[24] I. G. Niemegeers and M. Zafirovic-Vukotic, "HSLAN's for communication within a gateway: A performance evaluation," in Proc. IFIP WG 6.4 Workshop HSLAN'88, A. Danthine, Ed., Liege, North Holland, Apr. 1988, pp. $167-185$,

[25] L. D. Servi, "Average delay approximation of $M / G / 1$ cyclic service queues with Bernoulli schedules," IEEE J. Select. Areas Commun., vol. SAC-4, no. 6, pp. 813-822, 1986.

[26] C. E. Skinner, "A priority queueing system with server-walking time," Oper. Res., vol. 15, no. 2, pp 278-285, Mar.-Apr. 1967.

[27] H. Takagi, "Queueing analysis of polling models," ACM Comput. Surveys, vol. 20 , no. 1 , pp. 5-28, Mar. 1988.

[28] _ "Queueing analysis of vacation models, part I: M/G/1 and part II: $M / G / 1$ with vacations," TRL Res. Rep. TR87-0032, IBM Tokyo Res. Lab., Tokyo 102, 1987.

[29] S. Temple, "The design of the Cambridge fast ring," in Ring Technology Local Area Networks, I. N. Dallas and E. B. Spratt, Eds. Amsterdam, 
The Netherlands: Elsevier Sc.Pb. B. V., 1984, pp. 79-88.

[30] D. Valk, "Performance analysis of integrated services slotted ring protocols in gathering HSLANs," Rep. 080-87-08, Univ. Twente, Mar. 1987.

[31] E. Vyncke and A. Danthine, "A realistic simulation of a wideband backbone network," in Proc. ICCC'86, North Holland, 1986, pp. 176-18

[32] Z. D. Wu and E. B. Spratt, "The performance analysis of multiple Cambridge rings," in Proc. IFIP TC 6/WG6.4 Int. In-depth Symp. Local Commun. Syst.: LAN and PBX, J. P. Cabanel, G. Pujolle, and A. Danthine, Ed., Toulouse, North Holland, 1987.

[33] M. Zafirovic-Vukotic and I. G. Niemegeers, "An evaluation of high speed local area network access mechanisms," in Proc. Commun. Distributed Systems, Aachen, Informatik - Fachberichte no 130, SpringerVerlag, N. Gerner and O. Spaniol, Eds., Feb. 1987, pp. 426-440.

[34] , "Analytical models of the slotted ring protocols in HSLANs," in Proc. IFIP WG 6.4 Workshop High Speed Local Area Networks, A. Danthine, and O. Spaniol, Eds., Aachen, North Holland, 1987, pp. 115-134.

[35] __ "Performance modeling of the Orwell basic access mechanism," in Proc. ACM SIGCOMM'87: Frontiers in Comput. Commun. Technol., Stowe, Comput. Comm. Rev., Special Issue, vol. 17, no. 5, pp. 35-48, 1987.

[36] - "Performance modeling of a HSLAN slotted ring protocol," in Proc. ACM SIGMETRICS'88, Santa Fe, NM, Perform. Eval. Rev., Special Issue, vol. 16 , no. 1 , pp. 37-46, May 1988

[37] , "Performance modeling and analysis of the Orwell access mechanism," in Proc. ITC 12, Turin, Italy, June 1988, pp. 2.3B.5.1-2.3B.5.7.

[38] M. Zafirovic-Vukotic, I. G. Niemegeers, and D. Valk, "Performance analysis of slotted ring protocols in HSLANs," IEEE J. Select. Areas Commun., vol. SAC-6, no. 6, pp. 1011-1024, July 1988.
Mirjana Zafirovic-Vukotic received the B.S. degree in mathematics, minor in computer science, and the M.S. degree in operations research from the University of Belgrade, Belgrade, Yugoslavia, in 1981 and 1984, respectively, and the $\mathrm{Ph} . \mathrm{D}$. degree in engineering from the University of Twente, Enschede, Holland, in 1988.

She is currently a researcher at M.Pupin Institute, University of Belgrade, and an Associate Professor at the Electrical Engineering Faculty of the same university. Her current interests are in communication systems, performance modeling, and analysis.

Ignas G. Niemegeers (S'70-M'78) was born in Gent, Belgium, in 1947. He received the M.S. degree in electrical engineering from the Rijksuniversiteit Gent in 1970. In 1972 he received the M.S. degree in computer engineering and in 1978, the Ph.D. degree from Purdue University, West Lafayette, IN. From 1978 to 1981 he was a System Design Engineer at Bell Telephone Mfg. Cy., Antwerp, Belgium, where he was involved in the design of the DPS 1500 system, a high capacity X.25 packet switching network. From 1981 to 1986 he was with the Department of Electrical Engineering, University of Twente, Enschede, the Netherlands. Since 1986 he has been a Professor of computer science at the same university. His present research interests are architectures for local integrated communication infrastructures, parallel processing in communication switches, performance analysis, and performability of fault-tolerant systems. 